\title{
Luminescent Dipyrrinato Complexes of Trivalent Group 13 Metal Ions
}

\author{
Van S. Thoi, Jay R. Stork, Douglas Magde, * and Seth M. Cohen* \\ Department of Chemistry and Biochemistry, University of California, San Diego, La \\ Jolla, California, 92093-0358
}

scohen@ucsd.edu

\section{SUPPORTING INFORMATION}

RECEIVED DATE (to be automatically inserted after your manuscript is accepted if required according to the journal that you are submitting your paper to)

Group 13 Dipyrrinato Complexes

*Author to whom correspondence should be addressed. Telephone: (858) 822-5596.

Fax: (858) 822-5598. E-mail: scohen@ucsd.edu. 
Time Resolved Measurements. Fluorescence lifetimes were measured by timecorrelated photon counting (TCPC). A frequency-doubled, diode-pumped $\mathrm{Nd}^{3+}: \mathrm{VO}_{3}$ laser (Coherent, Verdi, 5 watts) pumped a home-built Ti:sapphire laser mode-locked by selfphase-modulation. The length of the cavity was adjusted to produce an interpulse spacing accurately equal to $12.0000 \mathrm{~ns}$, which then served as the time calibration for a histogram of counts versus time. Harmonic generation provided a train of excitation pulses near $400 \mathrm{~nm}$. A silicon photodiode monitored the excitation pulses and served as a time reference. Luminescence was collected with a fast, achromatic lens and spectrally resolved with a subtractive double monochromator (Spex 1672 Doublemate). Photons were detected with a microchannel plate PMT (Hamamatsu R1564-U01), amplified by a factor of ten (Phillips 774), and recognized by a constant-fraction discriminator (Tennelec TC454). The histogram for time delays was generated in reverse mode, in which a fluorescent photon started the "clock" (TAC, Canberra 2044) and the next available pulse from the excitation monitor stopped the "clock." The time interval, coded as a voltage pulse, was measured in a pulse height analyzer (Fastec MCA) at 6.565 ps per channel). Because the TAC can be paralyzed by a fast arrival of pulses, either starts or stops, a simple logic circuit inhibited stop pulses from reaching the TAC until a fluorescent photon was detected. The instrument response function (IRF), that is, the histogram of counts versus time for an instantaneous “delta function" photon emitter, was generated by scattering the excitation pulses using a dilute solution of coffee creamer in water. The change in the histogram between the IRF and a finite-lifetime emitter was analyzed by deconvolution, using iterative reconvolution, assuming a fit to a sum of exponentials. All software was written in house in order to minimize CPU processing time. A free 
parameter in all fits was a shift in the time origin. The shape of the IRF was quite constant, with occasional small, horizontal shifts in the time origin of less than $10 \mathrm{ps}$. Over the course of a day, half a dozen IRF were recorded. Each data set was deconvoluted using two, three, or more different IRF. Usually all fits gave identical lifetimes; occasionally there was a 10 ps variation. This established that results were independent of the IRF chosen and of any time shift in the origin.

Transient absorption measurements used excitation by a pulsed dye laser at $460 \mathrm{~nm}$ pumped by an $\mathrm{XeCl}$ laser, at about one pulse per second. Transient absorption and bleaching was measured one wavelength at a time with both continuous and pulsed probe lamps available. The probe propagated at right angles to excitation and its wavelength was defined by small monochromators before and after the sample along with lowemission glass filters. Detection used a high-current photomultiplier tube (Amperex TUVP56). The signal was digitized using a LeCroy 9361 oscilloscope. 
Table S1. Detailed fluorescence measurements on dipyrrinato complexes.

\begin{tabular}{|c|c|c|c|c|c|c|c|c|}
\hline \multicolumn{3}{|l|}{ Sample } & \multicolumn{2}{|l|}{ Tau } & \multicolumn{2}{|l|}{ lambda } & \multicolumn{2}{|c|}{ Goodness of fit } \\
\hline Compound & Solvent & Run & $500 \mathrm{~nm}$ & $530 \mathrm{~nm}$ & $560 \mathrm{~nm}$ & $590 \mathrm{~nm}$ & $\mathrm{X}^{2}$ & $\mathrm{D}-\mathrm{W}$ \\
\hline \multirow[t]{6}{*}{ Ga, prep 1} & Hexanes & Week 1 & & 3.73 & 3.73 & 3.73 & $1.1-1.5$ & $1.4-1.9$ \\
\hline & & Week 2 & 3.74 & 3.76 & 3.76 & 3.76 & $1.2-1.3$ & $1.5-1.7$ \\
\hline & & Week 3 & 3.76 & 3.77 & 3.76 & 3.75 & $1.05-1.08$ & $1.7-1.9$ \\
\hline & & Week 4 & 3.75 & 3.76 & 3.76 & 3.75 & $1.08-1.2$ & $1.7-1.9$ \\
\hline & МеСусHех & Week 1 & & 3.77 & 3.75 & 3.74 & $0.96-1.02$ & $1.9-2.0$ \\
\hline & & Week 2 & 3.74 & 3.75 & 3.73 & 3.75 & $1.1-1.1$ & $1.7-1.9$ \\
\hline \multirow[t]{7}{*}{ Ga, prep 2} & Hexanes & Week 1 & 3.74 & 3.75 & 3.75 & 3.74 & $1.07-1.2$ & $1.7-1.9$ \\
\hline & & Week 2 & & 3.73 & 3.71 & & $1.0-1.1$ & $1.8-1.9$ \\
\hline & & also & & 3.74 & & & 1.1 & 1.9 \\
\hline & MeCycHex & Week 1 & 3.76 & 3.77 & 3.76 & 3.76 & $1.1-1.2$ & $1.5-1.8$ \\
\hline & & Week 2 & 3.77 & 3.77 & 3.77 & 3.76 & $1.1-1.3$ & $1.5-1.8$ \\
\hline & & Week 3 & & 3.77 & 3.755 & & $1.1-1.2$ & $1.8-1.9$ \\
\hline & & also & & 3.76 & & & 1.1 & 1.9 \\
\hline \multirow[t]{7}{*}{ In } & Hexanes & Week 1 & 1.93 & 1.93 & 1.94 & 1.94 & $1.05-1.4$ & $1.4-1.8$ \\
\hline & & Week 2 & & 1.93 & 1.93 & & 1.2 & $1.7-1.9$ \\
\hline & & also & & 1.93 & & & 1.2 & 1.8 \\
\hline & MeCycHex & Week 1 & 1.79 & 1.80 & 1.80 & 1.79 & $1.1-1.3$ & $1.5-1.8$ \\
\hline & & Week 2 & 1.83 & 1.82 & 1.81 & 1.80 & $1.2-1.4$ & $1.5-1.7$ \\
\hline & & Week 3 & & 1.84 & 1.85 & & $1.5-1.6$ & $1.2-1.4$ \\
\hline & & also & & 1.84 & & & 1.4 & 1.4 \\
\hline \multirow[t]{4}{*}{$\mathrm{Zn}$} & Hexanes & Week 1 & 3.46 & 3.53 & 3.51 & 3.53 & & \\
\hline & & Week 2 & & $3.37^{a}$ & & & & \\
\hline & МeCycHex & Week 1 & 3.53 & 3.57 & 3.59 & 3.59 & & \\
\hline & & & & $3.62^{a}$ & & & & \\
\hline & & & & & & & & \\
\hline & & & & & & & & \\
\hline & & & & & & & & \\
\hline & & & & & & & & \\
\hline & & & & & & & & \\
\hline & & & & & & & & \\
\hline
\end{tabular}

${ }^{a}$ Best fit requires a very fast component in addition to this component. 
Figure S1. A drawing of the molecular structure of $[\mathrm{Ga}($ mesdpm $)] \cdot \mathrm{CDCl}_{3}$ with $50 \%$ thermal contours. Selected distances (A): Molecule 1: Ga1-N1, 2.054(2); $\mathrm{C} 1-\mathrm{C} 2, \quad 1.401(4) ; \mathrm{C} 1-\mathrm{N} 1,1.340(3) ;$ $\mathrm{C} 2-\mathrm{C} 3,1.371(4) ; \mathrm{C} 3-\mathrm{C} 4,1.424(3)$;


C4-C5, 1.400(3); C4-N1, 1.396(3); C5-C4A, 1.400(3); C5-C6, 1.500(5). Molecule 2 (not shown): Ga2-N2, 2.054(2); Ga2-N3, 2.055(2); C12-C13, 1.410(4); C12-N2, 1.339(3); C13-C14, 1.365(4); C14-C15, 1.432(4); C15-C16, 1.396(4); C15-N2, 1.393(3); C16-C17, 1.403(3); C16-C21, 1.505(4); C17-C18, 1.430(4); C17-N3, 1.396(3); C18-C19, 1.373(4); C19-C20, 1.409(4); C20-N3, 1.340(3). Selected angles (deg): Molecule 1: N1-Ga1-N1A, 89.04(12); N1-Ga1-N1B, 91.15(8); N1-Ga1-N1C, 91.15(8); N1-Ga1-N1D, 179.73(12); N1-Ga1-N1E, 88.65(12); C1-N1-C4, 106.0(2); N1-C1-C2, 111.9(2); C3-C2-C1, 106.3(2); C2-C3-C4, 107.2(2); N1-C4-C5, 124.5(2); N1-C4-C3， 108.5(2); C5-C4-C3，127.0(3); C4-C5-C4A，126.6(3); C4-C5-C6, 116.72(17). Molecule 2: N2-Ga2-N3, 89.07(8); C12-N2-C15, 106.5(2); N2-C12-C13, 115.5(2); C14-C13-C12, 106.6(2); C13-C14-C15, 107.1(3); N2-C15-C16, 125.0(2); N2-C15-C14， 108.3(2); C15-C16-C17，126.1(2); C15-C16-C21，116.6(2); C17-C16-C21，116.6(2); N3-C17-C16，124.8(2); N3-C17-C18，108.5(2); C16-C17-C18, 126.8(2); C19-C18-C17, 107.0(2); C18-C19-C20, 106.6(2); N3-C20-C19, 111.5(2); C20-N3-C17, 106.5(2). 
Figure S2. A drawing of the molecular structure of $[\operatorname{In}($ mesdpm $)] \cdot 0.25 \mathrm{MeCN}$ with $50 \%$ thermal contours. Selected distances (A): Molecule 1 (not shown): In1-N1, 2.212(3); C1-C2, 1.397(5); C1-N1, 1.335(4); C2-C3, 1.374(5); C3-C4,

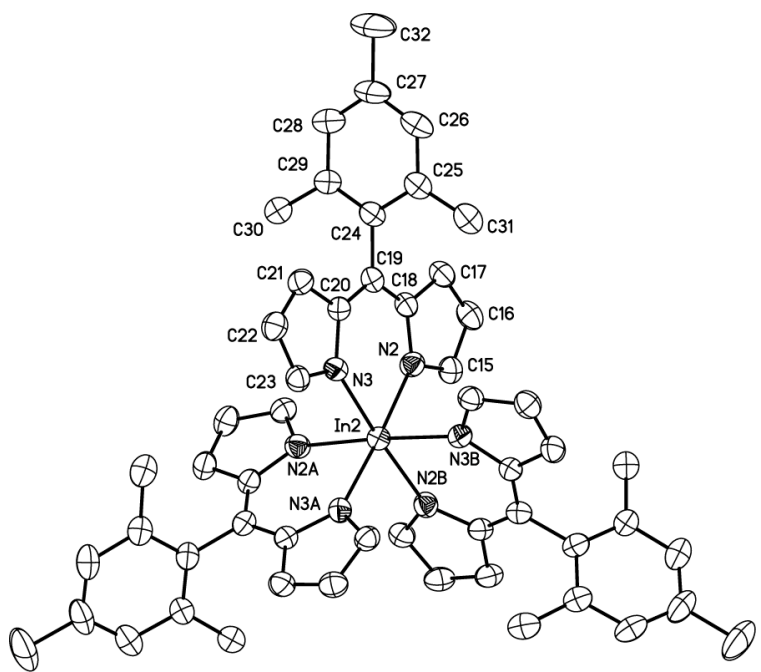
1.431(5); C4-C5, 1.402(4); C4-N1, 1.386(4); C5-C4A, 1.402(4); C5-C6, 1.515(7). Molecule 2: In2-N2, 2.219(3); In2-N3, 2.055(2); C15-C16, 1.406(6); C15-N2, 1.339(4); C16-C17, 1.374(5); C17-C18, 1.429(5); C18-C19, 1.407(5); C18-N2, 1.391(4); C19-C20, 1.400(5); C19-C24, 1.503(5); C20-C21, 1.433(5); C20-N3, 1.398(4); C21-C22, 1.378(5); C22-C23, 1.404(5); C23-N3, 1.336(4). Selected angles (deg): Molecule 1: N1-In1-N1A, 84.54(15); N1-In1-N1B, 93.28(10); N1-In1-N1C, 93.28(10); N1-In1-N1D, 176.96(15); N1-In1-N1E, 88.97(15); C1-N1-C4, 106.6(3); N1-C1-C2, 111.9(3); C3-C2-C1, 106.3(3); C2-C3-C4, 106.9(3); N1-C4-C5, 125.1(3); N1-C4-C3，108.3(3); C5-C4-C3，126.6(3); C4-C5-C4A，128.6(5); C4-C5-C6, 115.7(2). Molecule 2: N2-In2-N3, 84.08(10); C15-N2-C18, 106.7(3); N2-C15-C16, 111.5(3); C17-C16-C15, 106.3(3); C16-C17-C18, 107.2(3); N2-C18-C19, 125.5(3); N2-C18-C17，108.2(3); C18-C19-C20，127.4(3); C18-C19-C24，116.2(3); C20-C19-C24, 116.4(3); N3-C20-C19, 125.7(3); N3-C20-C21, 108.1(3); C19-C20-C21, 126.2(3); C22-C21-C20, 107.1(3); C21-C22-C23, 106.2(3); N3-C23-C22, 112.0(3); C23-N3-C20, 106.5(3). 




Figure S3. A drawing of the molecular structure of one of the two metal complexes in the asymmetric unit of $\left.\left[\mathrm{Ga}(4-\mathrm{cydpm})_{3}\right)\right] \cdot \mathrm{CHCl}_{3}$ with $50 \%$ thermal contours. Selected distances (̊̊): $\mathrm{Ga}(1)-\mathrm{N}(1), 2.043(2)$; $\mathrm{Ga}(1)-\mathrm{N}(8)$; $\mathrm{Ga}(1)-\mathrm{N}(2), 2.057(2) ; \mathrm{Ga}(1)-\mathrm{N}(4)$, 2.059(2); $\mathrm{Ga}(1)-\mathrm{N}(5), 2.066(2) ; \mathrm{Ga}(1)-\mathrm{N}(7), 2.054(2) ; \mathrm{Ga}(1)-\mathrm{N}(8), 2.034(2) ; \mathrm{C}(1)-\mathrm{N}(1)$, 1.330(4); $\mathrm{C}(1)-\mathrm{C}(2), 1.414(4) ; \mathrm{C}(2)-\mathrm{C}(3), 1.360(4) ; \mathrm{C}(3)-\mathrm{C}(4), 1.422(4) ; \mathrm{C}(4)-\mathrm{C}(5)$, 1.391(4); $\mathrm{C}(4)-\mathrm{N}(1), 1.403(3) ; \mathrm{C}(5)-\mathrm{C}(6), 1.391(4) ; \mathrm{C}(6)-\mathrm{N}(2), 1.403(3) ; \mathrm{C}(6)-\mathrm{C}(7)$, 1.424(4); C(7)-C(8), 1.370(4); C(8)-C(9), 1.406(4); C(9)-N(2), 1.333(3); C(5)-C(10), 1.496(4); C(21)-C(26), 1.489(4); C(37)-C(42), 1.501(4). Selected angles ( $\left.{ }^{\circ}\right)$ : N(1)Ga(1)-N(2), 88.69(9); N(4)-Ga(1)-N(5), 88.17(9); N(8)-Ga(1)-N(7), 89.11(9); C(1)-N(1)$\mathrm{C}(4), 105.9(2) ; \mathrm{N}(1)-\mathrm{C}(1)-\mathrm{C}(2), 112.2(3) ; \mathrm{C}(3)-\mathrm{C}(2)-\mathrm{C}(1), 105.9(3) ; \mathrm{C}(2)-\mathrm{C}(3)-\mathrm{C}(4)$, 107.8(3); N(1)-C(4)-C(3), 108.3(2); C(6)-C(5)-C(4), 126.9(3); N(2)-C(6)-C(7), 108.0(2); $\mathrm{C}(8)-\mathrm{C}(7)-\mathrm{C}(6), 107.5(3) ; \mathrm{C}(7)-\mathrm{C}(8)-\mathrm{C}(9), 106.2(3) ; \mathrm{N}(2)-\mathrm{C}(9)-\mathrm{C}(8), 111.8(3) ; \mathrm{C}(9)-$ $\mathrm{N}(2)-\mathrm{C}(6), 106.4(2)$. 


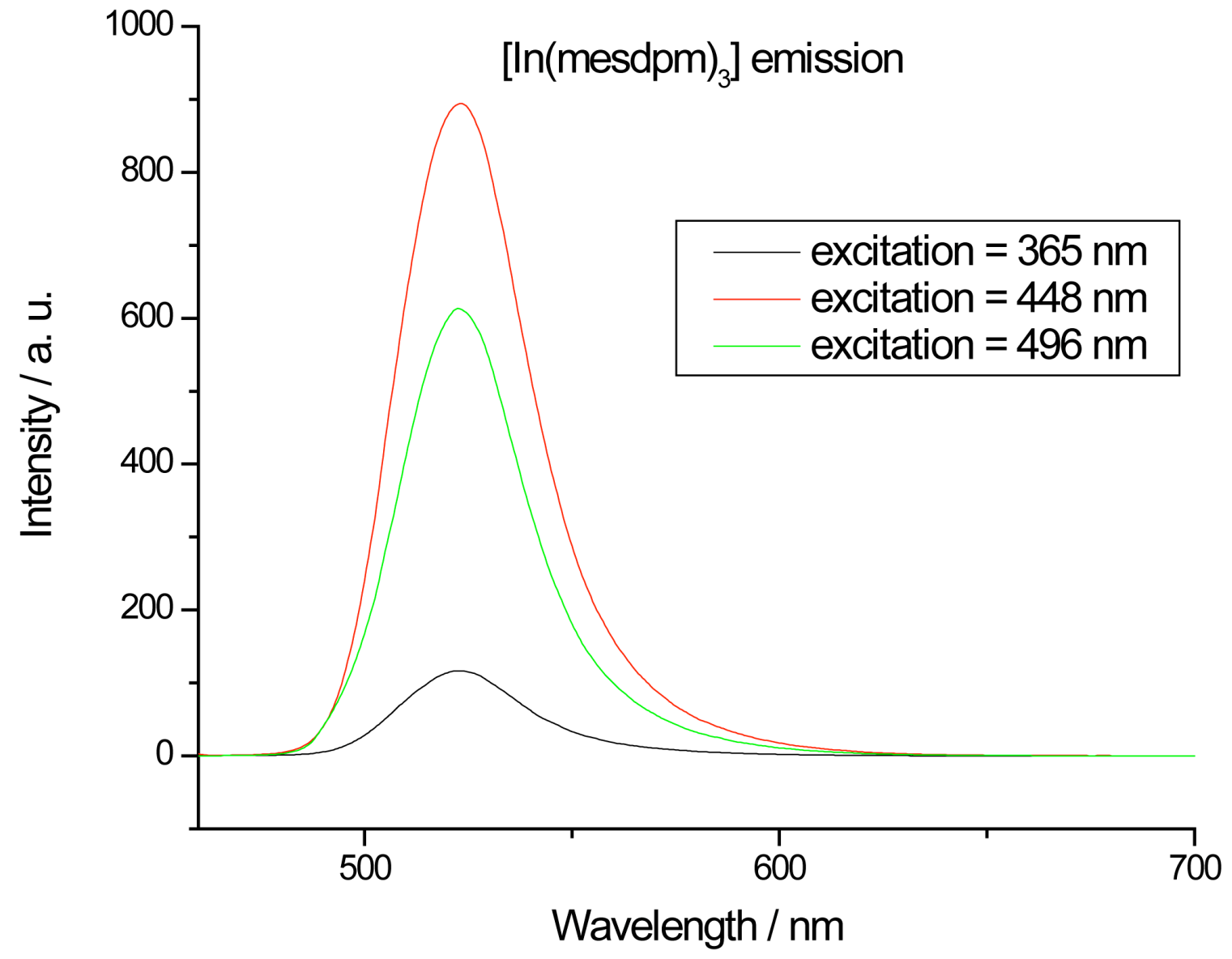

Figure S4. Emission of a $1 \times 10^{-6} \mathrm{M}$ solution of $\left[\operatorname{In}(\operatorname{mesdpm})_{3}\right]$ in hexanes excited at three different wavelengths. 


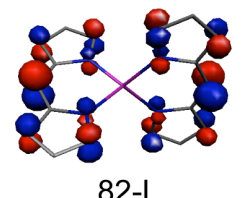

82-L

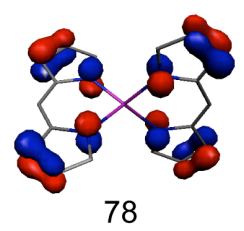

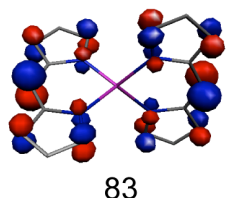

83

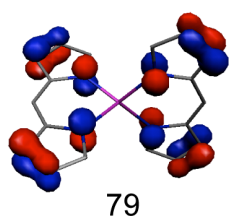

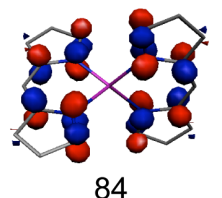

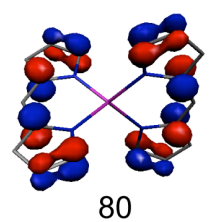

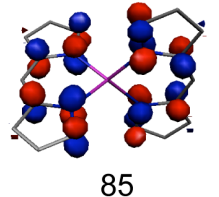

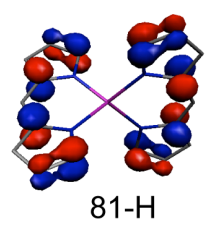

Figure S5. Drawings of the frontier orbitals calculated for [ $\left.\mathrm{Zn}(\mathrm{dpm})_{2}\right]$. 


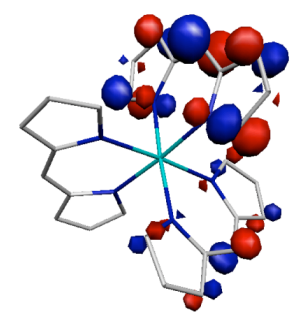

117

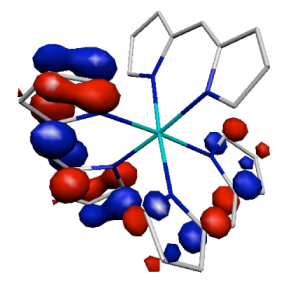

113

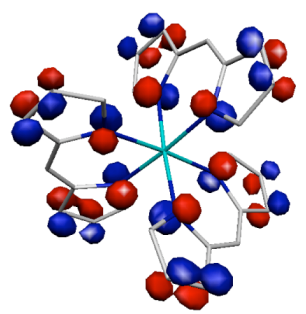

109

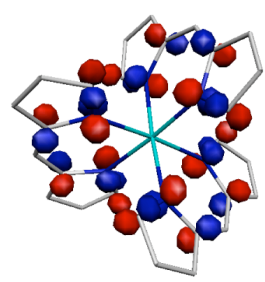

118

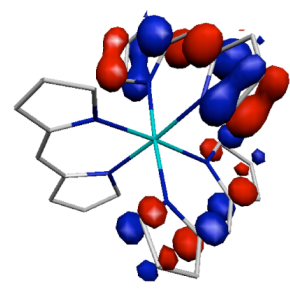

114-H

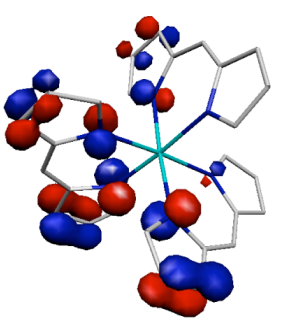

110

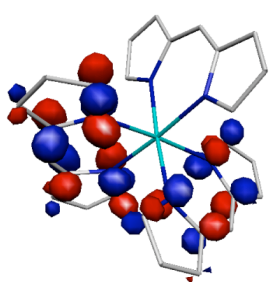

119

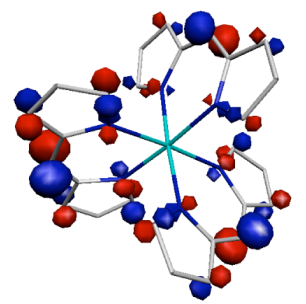

115-L

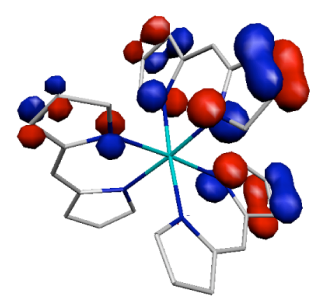

111

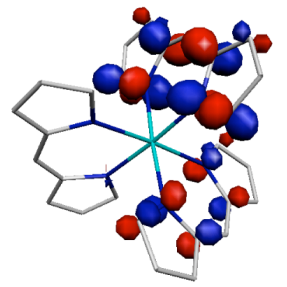

120

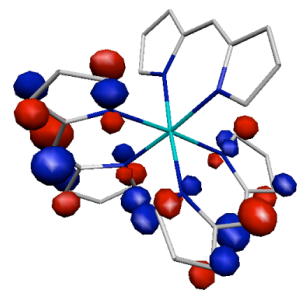

116

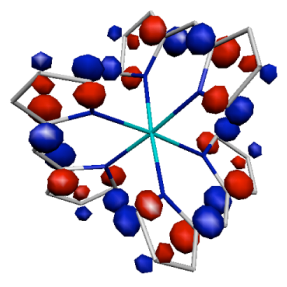

112

Figure S6. Drawings of the frontier orbitals calculated for $\left[\operatorname{In}(\mathrm{dpm})_{3}\right]$. 
Complete reference 28:

Frisch, M. J.; Trucks, G. W.; Schlegel, H. B.; Scuseria, G. E.; Robb, M. A.; Cheeseman, J. R.; Montgomery, J., J. A.; Vreven, T.; Kudin, K. N.; Burant, J. C.; Millam, J. M.; Iyengar, S. S.; Tomasi, J.; Barone, V.; Mennucci, B.; Cossi, M.; Scalmani, G.; Rega, N.; Petersson, G. A.; Nakatsuji, H.; Hada, M.; Ehara, M.; Toyota, K.; Fukuda, R.; Hasegawa, J.; Ishida, M.; Nakajima, T.; Honda, Y.; Kitao, O.; Nakai, H.; Klene, M.; Li, X.; Knox, J. E.; Hratchian, H. P.; Cross, J. B.; Bakken, V.; Adamo, C.; Jaramillo, J.; Gomperts, R.; Stratmann, R. E.; Yazyev, O.; Austin, A. J.; Cammi, R.; Pomelli, C.; Ochterski, J. W.; Ayala, P. Y.; Morokuma, K.; Voth, G. A.; Salvador, P.; Dannenberg, J. J.; Zakrzewski, V. G.; Dapprich, S.; Daniels, A. D.; Strain, M. C.; Farkas, O.; Malick, D. K.; Rabuck, A. D.; Raghavachari, K.; Foresman, J. B.; Ortiz, J. V.; Cui, Q.; Baboul, A. G.; Clifford, S.; Cioslowski, J.; Stefanov, B. B.; Liu, G.; Liashenko, A.; Piskorz, P.; Komaromi, I.; Martin, R. L.; Fox, D. J.; Keith, T.; Al-Laham, M. A.; Peng, C. Y.; Nanayakkara, A.; Challacombe, M.; Gill, P. M. W.; Johnson, B.; Chen, W.; Wong, M. W.; Gonzalez, C.; Pople, J. A. Gaussian 03, revision B.04, Gaussian, Inc.: Wallingford, CT, 2004. 